\title{
Investigation on risk factors of adult allergic rhinitis in China
}

\section{Guo Hong ( $\nabla$ goguo@163.com )}

Tianjin University of Traditional Chinese Medicine https://orcid.org/0000-0002-1350-4043

Zhou Yibo

Guangdong General Hospital: Guangdong Provincial People's Hospital

Li Fengxia

Tianjin University of Traditional Chinese Medicine

Liu Ming Ping

Guangzhou University of Traditional Chinese Medicine: Guangzhou University of Chinese Medicine

Cao Yong

Tianjin University of Traditional Chinese Medicine

\section{Research}

Keywords: Rhinitis/allergic, epidemiological survey, allergen spectrum, pathogenesis

Posted Date: May 10th, 2021

DOI: https://doi.org/10.21203/rs.3.rs-465698/v1

License: () (7) This work is licensed under a Creative Commons Attribution 4.0 International License. Read Full License 


\section{Abstract}

OBJECTIVE: To investigate the risk factors associated with allergic rhinitis in adults.

METHODS: Using the 1:1 matching principle, 1096 cases of allergic rhinitis patients aged 18-65 years were selected as the observation group, and another group of healthy medical checkups were selected as the control group, and the data related to allergic rhinitis were collected by allergen testing and questionnaire survey, and univariate and multifactorial analyses were performed respectively.

RESULTS: The univariate analysis showed that allergic rhinitis may be related to temperature changes, dryness and humidity, air pressure, wind speed, seasonal changes and exposure to allergens such as dust mites and house dust mites. After allergen testing of all patients in the observation group, it was found that dust mite and house dust mite were the highest percentage of allergens, accounting for $40.51 \%$, indicating that environmental factors play a decisive role in allergic rhinitis. Multi-factor analysis showed that exposure to dust mites, house dust mites, Penicillium punctatum, Streptomyces crossatus and Aspergillus fumigatus were independent risk factors for allergic rhinitis when it was humid, when it was cold, when it was windy and when the air pressure was low.

CONCLUSION: The epidemiological status of allergic rhinitis in adults in China was initially understood, and the main allergens were house dust mite and dust mite, which provided scientific epidemiological information for the standardized prevention and treatment of allergic rhinitis in this region.

\section{Background}

Allergic rhinitis is a chronic inflammatory disease of the nasal mucosa involving IgE antibodies in susceptible individuals after exposure to allergic allergens, initiated by the release of mediators (mainly histamine) from mast cells, and involving a combination of immunoreactive cells and cytokines. The prevalence of allergic rhinitis worldwide has been on the rise in recent years, and it has become a global health problem because it affects the quality of life of patients and increases the socioeconomic burden. With the increasing incidence of allergic rhinitis (AR) worldwide, related epidemiological studies have been intensifying, but in mainland China, a large country with a special geographical environment and a large number of patients, there are few epidemiological studies with overall representativeness, especially on the possible risk factors in the living environment and dietary habits of patients with allergic rhinitis. This study intends to investigate the possible risk factors in the living environment and dietary habits of patients with allergic rhinitis in mainland China through the collection and processing of risk factors in a large sample of normal and allergen-tested people with confirmed allergic rhinitis, and to provide a scientific basis for clinical monitoring, early intervention and control of allergic rhinitis.

\section{Case Presentation}

The purpose of this study is to observe the possible risk factors in the living environment and dietary habits of allergic rhinitis patients in mainland China, and to provide suggestions and guidance for the prevention and treatment of allergic rhinitis.A total of 1096 patients aged from 18 to 65 years were selected as the study subjects (all patients met the diagnostic criteria of 2015 Tianjin Guidelines of Otolaryngology Society of Chinese Medical Association). All the enrolled patients had no history of asthma,hypertension, coronary heart disease, psychosocial diseases, and did not receive any drug therapy during the observation period.Allergic rhinitis patients have nasal itching, sneezing, runny nose, nasal congestion and other symptoms, nasal mucosa pale, edema, nasal cavity with clear water like secretions.No other interventions were received, only blood samples were taken to check for allergens, and environmental risk factors for adult allergic rhinitis were analyzed.Results: Single factor analysis showed that allergic rhinitis might be related to temperature, wind speed, dry humidity, air pressure, seasonal change and exposure to house dust mites, dust mites and other allergens.After allergen detection for all patients with allergic rhinitis, it was found that house dust mites and dust mites accounted for the highest proportion of allergens, accounting for $40.51 \%$, indicating that environmental factors played a decisive role in allergic rhinitis.Multivariate analysis showed that exposure to house dust mites, dust mites, Mycospora penicillium, Aspergillus fumigatus and Streptospora were independent risk factors for allergic rhinitis in cold weather, high wind, humid weather and low air pressure.

Allergic rhinitis (AR) is a chronic inflammatory disease that afflicts approximately $10-30 \%$ of the population in the United States and is increasing in prevalence yearly, affecting approximately $40 \%$ of the world's population ${ }^{[1]}$. Allergic rhinitis is a component of systemic allergy and is associated with other allergic diseases, including asthma, sinusitis, and allergic conjunctivitis ${ }^{[2]}$. Allergic rhinitis reduces people's quality of life and affects sleep, learning, productivity and social life ${ }^{[2]}$. The impact of allergic rhinitis on quality of life has made it a class of respiratory diseases that urgently needs to be addressed ${ }^{[2]}$. In addition, the cost of treating allergic rhinitis is increasing year by year, and the medical costs of the disease now exceed those of diabetes, coronary heart disease, heart disease, and asthma ${ }^{[3]}$. It is thus crucial to identify the environmental risk factors for allergic rhinitis.

There are two main types of allergic rhinitis, namely seasonal (also known as intermittent) and perennial (also known as persistent) allergic rhinitis ${ }^{[4]}$. Symptoms of seasonal rhinitis are usually easily recognizable and directly related to exposure to seasonal allergens, such as grass, pollen, and fungi ${ }^{[1]}$. The periodicity of seasonal rhinitis can vary depending on geographic location and climatic conditions, and is also related to the duration of the patient's exposure to allergens ${ }^{[1]}$. Patients with intermittent rhinitis usually have episodes of less than four days per week or episodes of less than four weeks ${ }^{[2]}$. Symptoms of persistent allergic rhinitis usually occur approximately $75 \%$ of the time throughout the year, are greater than four days per week, last longer than four weeks, and are more difficult to diagnose due to the combination of other diseases such as sinusitis and respiratory infections ${ }^{[5]}$. Symptoms of persistent allergic rhinitis are usually caused by non-seasonal allergens such as mites, animal dander or molds.

The progression of allergic rhinitis is influenced by genetic and environmental factors ${ }^{[6]}$, especially environmental factors are the main cause of allergic rhinitis ${ }^{[7]}$. Numerous studies have found that environmental factors are the main causative factors of allergic rhinitis ${ }^{[6]}$. In this paper, a questionnaire survey 
will be conducted and allergen testing will be performed on 1096 patients with allergic rhinitis in China, in order to explore the possible risk factors in the living environment and dietary habits of patients with allergic rhinitis in China, and to provide suggestions and guidance for the prevention and treatment of allergic rhinitis.

\section{Object And Method}

\subsection{Subjects}

1096 patients diagnosed with allergic rhinitis in China City between November 2014 and July 2018, aged 18-65 years old, were selected for the study (the patients all met the diagnostic criteria of the 2015 Tianjin guidelines of the Chinese Medical Association, Ear, Nose and Throat Branch), and a homemade questionnaire was used to investigate and study 1096 patients with allergic rhinitis who met the diagnostic criteria and enrollment requirements, and to compare them with 1096 healthy individuals in the outpatient physical examination center during the same period as a control.

\subsection{Methods}

\subsubsection{Questionnaire}

The "clinical questionnaire for allergic rhinitis" was used, including whether the symptoms were aggravated when it was cold (the temperature was more than 20 degrees below the normal body temperature), when it was hot (the temperature was higher than the normal body temperature), and whether the symptoms were aggravated when it was hot.

Whether it is aggravated in spring, summer, autumn and winter, etc. To ensure the quality of the questionnaire, all questionnaires were completed under the supervision of the attending physician.

\subsubsection{Allergen detection}

The skin allergen prick test was performed. The skin prick test was performed using standardized prick antigens from ALK, Denmark. The antigens included house dust mite, dust mite, cockroach, cat fur dander, dog fur dander, Artemisia dwarf ragweed, Penicillium punctatum mycosporeum, Aspergillus fumigatus, Streptomyces crossatus, cypress, elm, sycamore, willow, trillium, humulus, egg, milk, fish, shrimp, crab, beef, mutton, cashew nut, peanut, soybean, mango, and wheat. Histamine dihydrochloride was used as a positive control and saline was used as a negative control.

\subsection{Statistical methods}

SPSS19.0 software was used to perform, and the chi-square test was used to compare the count data between groups, and multi-factor logistic regression analysis was used to screen the relevant risk factors.

\subsection{Informed consent and ethics}

All the selected candidates signed the informed consent and were discussed and approved by the ethics committee of the hospital.

\subsection{Results}

2.1 General There was no statistical significance between the two groups in terms of age composition, gender composition, race, climatic conditions and living environment $(P>0.05)$, and they were comparable.

\subsection{Climate and season and allergic rhinitis}

The questionnaire survey on climatic factors revealed that temperature changes, wind speed, dryness and humidity, and air pressure changes were all related to the aggravation of allergic rhinitis symptoms. After univariate analysis of 24 climatic and seasonal factors that may have an influence on the development of allergic rhinitis (see Table 1), it was found that the risk factors associated with the development of allergic rhinitis were: cold, windy, humid, low air pressure, and spring and autumn.

Table 1 Univariate analysis of risk factors for allergic minitis in 1096 cases 


\begin{tabular}{|c|c|c|c|c|c|c|c|c|c|c|c|c|c|c|c|c|c|c|c|}
\hline \multirow{3}{*}{ group } & \multicolumn{4}{|c|}{ Temperature change } & \multicolumn{4}{|c|}{ Wind Speed } & \multicolumn{4}{|c|}{ Dryness and humidity } & \multicolumn{4}{|c|}{$\begin{array}{l}\text { Barometric pressure } \\
\text { change }\end{array}$} & \multirow{2}{*}{\multicolumn{2}{|c|}{$\begin{array}{l}\text { Spring } \\
\text { aggravation }\end{array}$}} & \multirow{2}{*}{$\begin{array}{l}\text { Sum } \\
\text { aggri }\end{array}$} \\
\hline & \multicolumn{2}{|c|}{$\begin{array}{l}\text { Aggravated } \\
\text { when it is } \\
\text { cold }\end{array}$} & \multicolumn{2}{|c|}{$\begin{array}{l}\text { Aggravated } \\
\text { by the heat }\end{array}$} & \multicolumn{2}{|c|}{$\begin{array}{l}\text { aggravated } \\
\text { when windy }\end{array}$} & \multicolumn{2}{|c|}{$\begin{array}{l}\text { Wind hours } \\
\text { aggravated }\end{array}$} & \multicolumn{2}{|c|}{$\begin{array}{l}\text { Aggravated } \\
\text { when wet }\end{array}$} & \multicolumn{2}{|c|}{$\begin{array}{l}\text { Aggravated } \\
\text { when dry }\end{array}$} & \multicolumn{2}{|c|}{$\begin{array}{l}\text { aggravated } \\
\text { by high air } \\
\text { pressure }\end{array}$} & \multicolumn{2}{|c|}{$\begin{array}{l}\text { aggravated } \\
\text { by low air } \\
\text { pressure }\end{array}$} & & & \\
\hline & yes & no & yes & no & yes & no & yes & no & yes & no & yes & no & yes & no & yes & no & yes & no & yes \\
\hline $\begin{array}{l}\text { Allergic } \\
\text { rhinitis } \\
\text { group }\end{array}$ & 609 & 487 & 307 & 789 & 458 & 638 & 120 & 976 & 649 & 447 & 335 & 761 & 227 & 869 & 758 & 338 & 367 & 729 & 117 \\
\hline $\begin{array}{l}\text { Healthy } \\
\text { control } \\
\text { group }\end{array}$ & 156 & 940 & 33 & 1063 & 34 & 1062 & 28 & 1068 & 87 & 1009 & 28 & 1068 & 40 & 1056 & 129 & 967 & 50 & 1046 & 39 \\
\hline$c^{2 \text { values }}$ & 8.111 & & 1.145 & & 17.5 & & 3.092 & & 10.6 & & 3.077 & & 2.91 & & 5.61 & & 3.307 & & 7.13 \\
\hline Pvalues & 0.019 & & 0.158 & & 0.03 & & 0.087 & & 0.00 & & 0.08 & & 0.074 & & 0.045 & & 0.001 & & 0.06 ? \\
\hline
\end{tabular}

\subsection{Allergens and allergic rhinitis}

In order to understand the relationship between allergens and allergic rhinitis, allergen testing was performed on 1096 patients with allergic rhinitis, and it was found that house dust mite and dust mite accounted for the highest proportion of environmental factors, reaching $40.51 \%$, and were the most important allergens for allergic rhinitis. The environmental factors followed by Penicillium punctatum, Aspergillus fumigatus, Streptomyces crossatus, accounting for $15.43 \%$. And among the dietary factors, fish, shrimp and crab were the major allergens of allergic rhinitis, accounting for $5.38 \%$ (Table 2 ). These results indicate that house dust mite and dust mite among environmental factors are the main allergens of allergic rhinitis and play a major role in the development of allergic rhinitis.

\section{Table 2 Allergen detection results of 1096 cases of allergic rhinitis}

\begin{tabular}{|lll|}
\hline Name & Number of cases & Composition ratio $\%$ $\square$ \\
\hline Dust particles, powder dust particles & 444 & 40.51 \\
\hline Cockroach & 108 & 9.85 \\
\hline Cat fur dander, dog fur dander & 82 & 7.48 \\
\hline Artemisia dwarf ragweed & 126 & 11.49 \\
\hline Penicillium punctatum Mycosphaerella, Aspergillus fumigatus, Streptomyces crossatus & 169 & 15.43 \\
\hline Cypress, elm, sycamore, willow, triangular-leaved poplar & 38 & 3.47 \\
\hline Humulus japonicus & 32 & 2.92 \\
\hline Eggs & 5 & 0.46 \\
\hline Milk & 4 & 0.36 \\
\hline Fish, shrimp and crab & 59 & 5.38 \\
\hline Beef, lamb & 6 & 0.55 \\
\hline Cashew nuts, peanuts, soybeans & 3 & 0.27 \\
\hline Mango & 1 & 0.09 \\
\hline Wheat & 0 & 0 \\
\hline Combining two or more allergens & 19 & 1.74 \\
\hline
\end{tabular}

\subsection{Multi-factor analysis of environment-related risk factors for allergic rhinitis}

Multi-factor logistic regression analysis was performed and significant results are shown in Table 3. In terms of OR, house dust mite, dust mite (OR=9.458,95\% Cl: 4.011 19.373), Penicillium punctatum Mycosphaerella, Aspergillus fumigatus, Streptomyces crossatus (OR=,8.024, 95\%Cl:3.677 17.211), aggravated by humidity ( $\mathrm{OR}=2.797,95 \% \mathrm{Cl}: 1.862-4.233)$, when windy (OR=2.015,95\%Cl: 1.311-3.048), when cold (OR=1.673,95\%Cl: 1.127-2.529), and when air pressure was low $(\mathrm{OR}=1.039,95 \% \mathrm{Cl}: 1.012-1.059)$. The risk of allergic rhinitis was higher for allergic rhinitis $(\mathrm{OR}=1.039,95 \% \mathrm{Cl}$ : 1.012 1.059).

Table 3 Multifactorial analysis of environment-related risk factors for allergic rhinitis in China 


\begin{tabular}{|lllll|}
\hline Influencing Factors & OR & $95 \% \mathrm{Cl}$ & & $\mathrm{P}[$ \\
\cline { 3 - 5 } & & Lower & Upper & \\
Aggravated when it is cold & 1.673 & 1.127 & 2.529 & 0.042 \\
\hline aggravated when windy & 2.015 & 1.311 & 3.048 & 0.049 \\
\hline Aggravated when wet & 2.797 & 1.862 & 4.233 & 0.037 \\
\hline aggravated by low air pressure & 1.039 & 1.012 & 1.059 & 0.038 \\
\hline Dust particles, powder dust particles & 9.458 & 4.011 & 19.373 & 0.001 \\
\hline Penicillium punctatum Mycosphaerella, Aspergillus fumigatus, Streptomyces crossatus & 8.024 & 3.677 & 17.211 & 0.029 \\
\hline
\end{tabular}

\section{Discussion}

In this paper, we found that cold, windy, humid, low air pressure and spring and autumn were all environmental risk factors for allergic rhinitis in 1096 patients with allergic rhinitis. In the allergen analysis, house dust mite and dust mite were found to account for the highest proportion of all allergens, indicating that the living environment plays an important role in the development of allergic rhinitis. In addition, diet also plays an important role in allergic rhinitis, and people who are allergic to fish, shrimp and crab are also those who have a higher incidence of allergic rhinitis. Our results suggest that strengthening the cleanliness of the home environment, minimizing the density of dust mites in the air, paying attention to a reasonable dietary structure, and paying attention to the climate and seasonal changes have clinical significance in reducing the incidence of allergic rhinitis.

\subsection{Climatic and seasonal factors}

There is evidence that climatic factors may indirectly cause the symptoms of allergic rhinitis, but do not necessarily increase the prevalence of allergic rhinitis. A recent study found that the prevalence of allergic rhinitis in the United States varied significantly by region and was associated with various climatic factors, including humidity, drought, temperature, and precipitation ${ }^{[7]}$. The highest prevalence was found in the southeastern and southern states, where both humidity and temperature are higher. Other studies have shown that global temperature changes may affect the severity of allergic rhinitis through to pollen and air. For example, temperature changes lead to a longer duration of the pollen season and thus an increase in the level of pollen allergy ${ }^{[8]}$. International Phenology has observed for almost 30 years that spring is six days earlier than in the past and the duration of the pollen season is prolonged, making the exposure of susceptible people to allergens longer, which in turn exacerbates allergic reactions ${ }^{[9]}$. Pollen is not the only climatic factor that increases the prevalence of allergic rhinitis. Temperature and humidity likewise contribute to the late metaplastic response of eosinophils ${ }^{[10]}$. In addition, warm climate shows a positive correlation with allergic rhinitis ${ }^{[11]}$. One study predicts that by 2050, the average temperature will increase by 3 degrees Celsius and thus the number of patients with allergic rhinitis due to pollen will increase to $40 \%{ }^{[12]}$. Therefore, changes in climate may be a major factor in the increasing prevalence of allergic rhinitis ${ }^{[13]}$. In this paper, we found that changes in temperature, wind speed, dryness and humidity, and changes in air pressure were associated with increased symptoms of allergic rhinitis. Cold, windy, humid and low air pressure are all environmental risk factors for allergic rhinitis. In addition, the highest percentage of allergic rhinitis symptom exacerbation was found in spring and autumn, indicating that spring and autumn are also environmental risk factors for allergic rhinitis.

\subsection{Allergens}

An increase in the prevalence of allergic rhinitis has been shown to be associated with increased exposure to indoor allergens due to changes in housing and lifestyle ${ }^{[14]}$. Exposure to susceptible allergens is a major cause of the development of allergic rhinitis. Certain allergens, such as those from dust mites, cockroaches, molds, and pollen are linearly associated with the development of allergic rhinitis. The duration of exposure to allergens is a key factor in allergic rhinitis ${ }^{[15]}$. Increased concentration of indoor dust mite allergens is associated with the risk of allergic rhinitis. It has been noted that indoor temperature, humidity and living environment lead to increased exposure to indoor allergens, especially dust mites, and allergic rhinitis ${ }^{[16]}$. Elevated indoor temperatures, walls or carpets contribute to enhanced levels of dust mite allergens and are therefore associated with sensitivity to allergic rhinitis ${ }^{\text {[17] }}$. House dust mites are the most common dust mites found indoors and are the main allergens of allergic diseases such as allergic rhinitis. Studies have shown that Dermatophagoides pteronyssinus and Dermatophagoides Felina are the most common mites in the world. House dust mites are commonly found in products such as bedding, carpets, curtains and fabrics. House dust mite hypersensitivity is closely associated with persistent allergic rhinitis ${ }^{[18]}$. Avoiding exposure to allergens, including house dust mites, is the main treatment strategy for allergic rhinitis and asthma [3]. In this paper, the highest proportion of house dust mites and dust mites among environmental factors was found to be the most important allergens in allergic rhinitis in 1096 patients with allergic rhinitis, reaching $40.51 \%$. Thus, reducing exposure to house dust mites indoors and dust mites outdoors is the main means to reduce the occurrence of allergic rhinitis.

In addition, mold exposure is also associated with allergic rhinitis. Indoor humidity and mold exposure are considered potential risk factors for allergic rhinitis. Several studies have found a strong association between mold exposure and childhood asthma ${ }^{[9]}$. A 2013 retrospective analysis examined the association between mold exposure and allergic rhinitis and found that exposure to dampness and mold was associated with allergic reactions including allergic rhinitis [4]. These results suggest that preventing indoor dampness and reducing mold may reduce the risk of developing allergic rhinitis. The analysis of environmental risk factors in patients with allergic rhinitis in this paper helps to correctly understand the environmental risk factors for allergic rhinitis and provides a theoretical basis for the prevention and treatment of allergic rhinitis. 


\section{Declarations}

1. Ethics approval and consent to participate

Ethical approval and consent to participateAnswer:The five authors of this paper submitted ethical review to the ethics committees of their four institutions, which are named as follows: the ethics committee of Tianjin Binhai New Area Hospital of Traditional Chinese Medicine, the ethics committee of Guangdong Second People's Hospital, the ethics committee of Guangzhou University of Traditional Chinese Medicine College of Pharmacy, and the ethics committee of Jinan University College of Traditional Chinese Medicine, all four committees considered that there was no intervention in the project and that the patients did not require ethical review, and no approval document for ethical review was issued, requiring us to submit only ethical statements and presentations to $\mathrm{SCl}$ journals. All observers and healthy controls signed informed consent forms. Consent to publicationAnswer:All authors have given their consent for you to publish our paper.

2. Consent for publication

All of our authors agree to publish in your journal Allergy, Asthma \& Clinical Immunology

3. Availability of data and materials

We guarantee that all the data and materials involved in this article are true and reliable

4. Competing interests

We have no competing interests, especially commercial interests, among our authors or with other peers. And as explained in the submitted "Conflict of Interest Statement"

5. Funding

This article did not receive any funding

6. Authors' contributions

The authors' contributions are: Guo Hong first, Zhou Yibo second, Li Fengxia third, Liu Mingping fourth, and Cao Yong fifth

7. Acknowledgements

Not applicable

\section{References}

1. Okubo K. Kurono Y, Ichimura K,et al. Japanese guidelines for allergic rhinitis 2020. Allergol Int. 2020 Jul;69(3):331-345.

2. Rosario CS. Murrieta-Aguttes M, Rosario NA. Allergic rhinits: impact on quality of life of adolescents. Eur Ann Allergy Clin Immunol. 2020 Nov 12. doi: 10.23822/EurAnnACl.1764-1489.176. Epub ahead of print. PMID: 33182991.

3. Thanborisutkul K. Khodtecha N, Kulalert P, et al Efficacy, patients' perception, and cost of medication in allergic rhinitis with subcutaneous immunotherapy. Asian Pac J Allergy Immunol. 2020 Aug 23. doi: 10.12932/AP-040320-0781.

4. Dykewicz MS. Wallace DV, Amrol DJ, et al; Rhinitis 2020: A practice parameter update. J Allergy Clin Immunol 2020 Oct;146(4):721-67.

5. Bousquet J. Anto JM, Bachert C, et al Allergic rhinitis. Nat Rev Dis Primers. 2020 Dec 3;6(1):95.

6. Meirlaen L. Levy El, Vandenplas Y. Prevention and Management with Pro-, Pre and Synbiotics in Children with Asthma and Allergic Rhinitis: A Narrative Review. Nutrients. 2021 Mar 14;13(3):934.

7. Nkosi V. Rathogwa-Takalani F, Voyi K. Non-allergic rhinitis and associated risk factors among the elderly in communities close to gold mine dumps in Gauteng and North West provinces in South Africa: a cross-sectional study. Int Arch Occup Environ Health. 2020 Aug,93(6):715-21.

8. Hou H. Cui X, Xu H, et al Quality of life survey of patients with allergic rhinitis caused by airborne pollen in Inner Mongolia. Lin Chung Er Bi Yan Hou Tou Jing Wai Ke Za Zhi. 2020 May,34(5):421-424. Chinese.

9. Di Cicco ME, Ferrante G. Amato D, et al. Climate Change and Childhood Respiratory Health: A Call to Action for Paediatricians. Int J Environ Res Public Health. 2020 Jul 24;17(15):5344.

10. Kordit DS. Reznik M, Leu CS, et al Longitudinal trends in asthma emergency department visits, pollutant and pollen levels, and weather variables in the Bronx from 2001-2008. J Asthma. 2020 May;57(5):487-94.

11. Eguiluz-Gracia I. Mathioudakis AG, Bartel S, et al The need for clean air: The way air pollution and climate change affect allergic rhinitis and asthma. Allergy 2020 Sep;75(9):2170-84.

12. Pfaar O. Karatzas $\mathrm{K}, \mathrm{Bastl} \mathrm{K}$,et al. Pollen season is reflected on symptom load for grass and birch pollen-induced allergic rhinitis in different geographic areas-An EAACI Task Force Report. Allergy 2020 May;75(5):1099-106.

13. Todkill D. de Jesus Colon Gonzalez F, Morbey R, et al. Environmental factors associated with general practitioner consultations for allergic rhinitis in London, England:a retrospective time series analysis. BMJ Open.2020 Dec 4;10(12):e036724. 
14. Bergmann KC. Krause L, Hiller J, et al First evaluation of a symbiotic food supplement in an allergen exposure chamber in birch pollen allergic patients. World Allergy Organ J. 2020 Dec 18;14(1):100494.

15. Huang CF. Chie WC, Wang IJ. Effect of environmental exposures on allergen sensitization and the development of childhood allergic diseases: $A$ largescale population-based study. World Allergy Organ J. 2021 Jan 6;14(1):100495.

16. Berson SR. Klimczak JA, Prezio EA, et al House Dust Mite Related Allergic Rhinitis and REM Sleep Disturbances.Am J Otolaryngol.2020 NovDec,41(6):102709.

17. Huang C. Zhang J, Sun C, et al Associations between household renovation and rhinitis among preschool children in China: A cross-sectional study. Indoor Air. 2020 Sep,30(5):827-40.

18. Kim YJ. Lee MY, Yang AR, et al Trends of Sensitization to Inhalant Allergens in Korean Children Over the Last 10 Years. Yonsei Med J.2020 Sep;61(9):797804. 